\title{
Startschuss für «DermaCampus» - scharfe Inhalte für weitsichtige Leser
}

Mit dieser Ausgabe von Karger Kompass Dermatologie stellen wir Ihnen erstmals den «DermaCampus» vor - eine Rubrik, die wir dezidiert den Belangen junger Fachärzte und Weiterbildungsassistenten widmen. Wir geben der jungen Ärzteschaft damit eine Plattform, um ihre Anliegen zu formulieren, aber auch um Wissen zu vermitteln und Hilfestellung in beruflichem und wissenschaftlichem Kontext zu geben: Zu Wort kommen die Jungmediziner in fachspezifischen Darstellungen, berufspolitischen Auseinandersetzungen sowie mit aktuellen Projektvorstellungen.

In der ersten Ausgabe des Jahres 2017 finden Sie einen Beitrag von Dr. Sven Riestenpatt, Projektmanager und Lektor des Karger Verlags in Freiburg, zur Frage «Wie schreibe ich einen wissenschaftlichen Artikel?». Damit leiten wir eine dreiteilige Serie zum Thema des wissenschaftlichen Publizierens ein, die Ihnen klar und überschaubar den Schritt vom Experiment bzw. der Studie zur Publikation erleichtern wird. Anschließend spricht Dr. Kevin Schulte, Sprecher des Bündnis JUNGE ÄRZTE, in einem Interview über die Perspektiven und Herausforderungen junger Ärztinnen und Ärzte in Deutschland. Er geht dabei nicht nur auf die Probleme der Jungmediziner ein, sondern erläutert auch, was es braucht, um diese zu lösen und warum es sich lohnt, positiv in die Zukunft zu blicken.

Die KaRger Kompass-Redaktion wünscht Ihnen viel Freude beim Lesen und ist jederzeit offen für Fragen und dankbar für Ihre Kritik oder Anregungen!

Redaktion Karger Kompass, Karger Verlag Freiburg 
Irgendwann ist es soweit. Alle Experimente sind gemacht, die Rohdaten sind ausgewertet, die Schlussfolgerungen sind gezogen. Nun steht der nächste Schritt an: das Schreiben eines wissenschaftlichen Papers und dessen Publikation. Dies sei vorausgeschickt: Das Schreiben eines wissenschaftlichen Artikels ist harte Arbeit!

\section{Vorbereitung}

Zunächst sollte man sich klar machen, worüber und was man schreiben will. Sehr hilfreich sind hier die FINER-Kriterien:

- $\quad$ Feasible = machbar

- $\quad$ Interesting = interessant

- $\quad$ Novel = neu

- $\quad$ Ethical = in Übereinstimmung mit ethischen Grundsätzen

- $\quad$ Relevant = relevant.

Ist eines dieser Kriterien nicht erfüllt, sinken die Chancen für eine Publikation dramatisch.

Schon während der experimentellen Phase sollte man kontinuierlich sein Studiendesign hinterfragen und gegebenenfalls anpassen und systematische Fehler korrigieren bzw. ausschließen. Ebenso müssen ethische Fragen schon im Vorfeld geklärt sein. Liegt die Zustimmung des Ethik-Komitees vor? Sind die Patienten informiert worden und haben sie ihre Zustimmung gegeben? Ist die Vertraulichkeit der Patientendaten sichergestellt? Sind konkurrierende Interessen (z.B. Firmensponsoring) deklariert?

Darüber hinaus ist zu empfehlen, sich bei der statistischen Auswertung beraten zu lassen.

\section{Durchführung}

- Zeitig beginnen - ein wesentlicher Teil des Artikels (z.B. die Einleitung und der Material-und-Methoden-Teil) kann bereits vorbereitet werden, auch wenn diese Teile eventuell später noch angepasst werden müssen.

- Klar und deutlich schreiben - «keep it simple», vermeiden Sie Laborjargon und Abkürzungen, seien Sie genau und konkret. Achten Sie besonders auf Elemente Ihres Artikels, die hohe

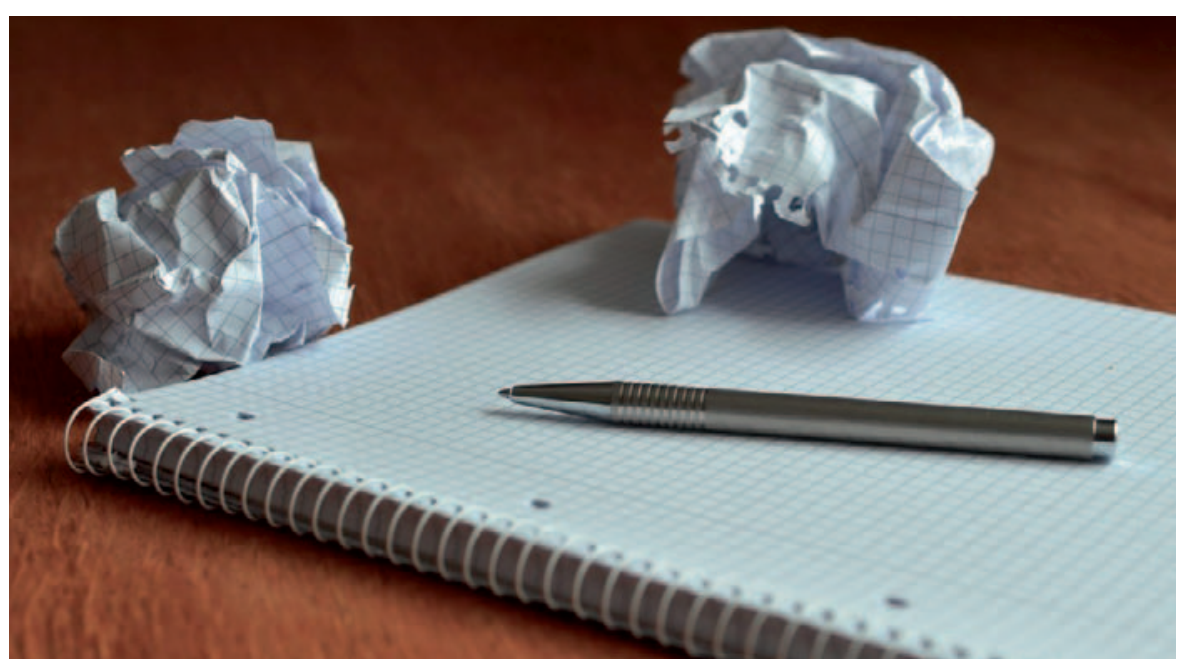

Aufmerksamkeit auf sich ziehen, z.B. Titel, Abstract, Schlussfolgerung sowie Abbildungen und Tabellen.

- Strukturieren Sie Ihren Artikel - Ihr Artikel ist leichter zu lesen, wenn Sie Unterüberschriften nutzen und auf eine konsequente Verwendung von Bezeichnungen achten.

- $\quad$ Entwickeln Sie eine systematische Struktur

- Einleitung: Warum stellt sich diese wissenschaftliche Frage?

- Methoden: Was habe ich gemacht?

- Ergebnisse: Was habe ich herausgefunden?

- Diskussion: Was bedeuten diese Ergebnisse?

- Zitieren Sie alle im Artikel verwendeten Quellen (beachten Sie die Hinweise für Autoren des jeweiligen Journals in Hinblick auf die Formatierung der Referenzen).

- Verwenden Sie eine strukturierte Literaturdatenbank-Abfrage (z.B. PubMed), um Ihre Literaturliste auf dem neuesten Stand zu halten.

- Bitten Sie erfahrene Kollegen um ein Feedback und um Anregungen zur Verbesserung Ihres Artikels.

\section{Abschluss}

Wählen Sie ein geeignetes Journal (Ausrichtung der Zeitschrift, Renommee) aus. Merke: Nicht jeder Artikel eignet sich für eine Publikation in Nature. Ihr Artikel muss von der Thematik her zum Journal passen und dessen wissenschaftlicher Stellenwert sollte ich sich ebenfalls mit den von dieser Zeitschrift publizierten Artikeln decken. Holen Sie sich auch hier Unterstützung von erfahrenen Kollegen. Prüfen Sie Ihren Artikel nochmals in Hinblick darauf, ob die in den Hinweisen für Autoren des ausgewählten Journals angegebenen formalen Kriterien erfüllt sind. Eine Nichtbeachtung dieser Kriterien kann schon zur Ablehnung des Artikels führen, ohne dass er weiter geprüft werden würde. Beachten Sie Vorgaben des ausgewählten Journals zur Einreichung von Artikeln. Die meisten wissenschaftlichen Zeitschriften verwenden heute Online-Einreichungssysteme, die über die Homepage des jeweiligen Journals zu erreichen sind.
Empfohlene weiterführende Literatur International Committee of Medical Journal Editors: Uniform requirements for manuscripts submitted to biomedical journals. www.icmje.org (Zugriff 16.01.17). Welch HG: Preparing manuscripts for submission to medical journals: the paper trail. http:// ecp.acponline.org/mayjun99/welch.htm (Zugriff 16.01.17).

Groves T: Fundamentals of good medical writing. http://docslide.us/documents/ fundamentals-of-good-medical-writing.html (Zugriff 16.01.17).

Kontaktadresse: Dr. Sven Riestenpatt, Projektmanagement \& Editorial Office Transfusion Medicine and Hemotherapy / Obesity Facts, S. Karger Verlag für Medizin und Naturwissenschaften $\mathrm{GmbH}$, Wilhelmstraße 20a, 79098 Freiburg, Deutschland, s.riestenpatt@karger.com. 


\section{Bündnis JUNGE ÄRZTE}

\section{Zwischen Patientenversorgung und Selbstmanagement - Herausforderungen und Perspektiven guter ärztlicher Arbeit}

Dr. med. Kevin Schulte ist Sprecher des Bündnis JUNGE ÄRZTE und Sprecher der außerordentlichen Mitglieder im Vorstand des Berufsverbandes Deutscher Internisten e.V. (BDI). Seit 2015 ist er als Assistenzarzt an der Klinik für Nieren- und Hochdruckkrankheiten des Universitätsklinikums Schleswig-Holstein, Kiel tätig. Karger KompaSS hat inn in seiner Funktion als Sprecher des Bündnis JUNGE ÄRZTE unter anderem zu den Herausforderungen junger Mediziner in Weiterbildung und Beruf sowie zu seinen Ansätzen für eine gelungene medizinische Versorgung befragt.

Was war der Antrieb zur Gründung des Bündnis JUNGE ÄRZTE? Welche Ziele verfolgt das BJÄ?

Wir, also die Sprecher/innen der Weiterzubildenden der 20 größten deutschen Berufs- und Fachverbände, hatten den Eindruck, dass wir, unabhängig von unserer Fachdisziplin, mit denselben alltäglichen Problemen konfrontiert sind. Daher war der Gedanke für uns naheliegend: Lasst uns gemeinsame Sache machen und zusammen an guten Lösungen arbeiten.

Mit welchen Herausforderungen sehen sich Assistenzärzte konfrontiert?

In den letzten 10 Jahren hat sich in deutschen Krankenhäusern viel verändert: Zunehmend ältere und kränkere Patienten werden in immer kürzerer Zeit mit komplexeren Behandlungsmethoden behandelt. Diese Tendenz stellt nicht nur unsere Patient/innen vor eine große Herausforderung, sondern auch uns als die behandelnden Ärzt/innen. Weiterhin hat sich unsere Vorstellung davon verändert, wie Arbeit in ei-

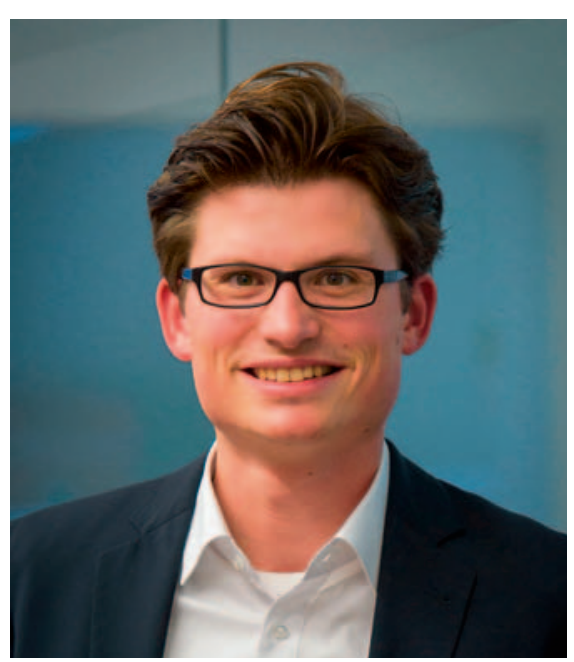

«Wir brauchen wieder

Lust auf Forschung, Fortschritt und Veränderung!»
Betriebskosten basieren, ihre Investitionsmittel zu erwirtschaften. Das geht nur, wenn die Kliniken billiger arbeiten als die InEK-Kalkulationshäuser - ein absurder Zustand. Wichtig ist: Die aktuelle Situation führt dazu, dass wirtschaftliche Motive unsere Patienten aus dem Mittelpunkt unseres ärztlichen Handels verdrängen. Das ist falsch und unethisch. Ähnlich hat sich hierzu übrigens kürzlich der deutsche Ethikrat positioniert.

Die Fachgesellschaften und Kliniken buhlen um junge Ärzte. Welche Argumente überzeugen? Mit welchen Argumenten kann die Richtungsentscheidung beeinflusst werden?

Das beste Argument ist gute Arbeit! Also für die Kliniken: gute Patientenversorgung, professionelles Personalmanagement und ein respektvolles Miteinander. Für die Berufs- und Fachgesellschaften: engagierte Interessensvertretung und gute Fortbildungen. Leere Versprechungen und Werbekugelschreiber sind absolut verzichtbar!

In der Umfrage des Marburger Bundes von 2015 klagen 59\% der befragten Klinikärzte über häufige psychische Belastung, $69 \%$ geben an, nicht genügend Zeit für die Patientenversorgung zu haben. Zudem kommt das Problem der Vereinbarkeit von Familie und Beruf. Welche Strukturreformen halten Sie in der Medizin für unumgänglich, um den Arbeitsplatz Krankenhaus wieder attraktiv werden zu lassen?

Wir brauchen sowohl im Beruflichen als auch im Privaten einen stärkeren Fokus auf das, was nur von uns getan werden kann und uns wichtig ist. Also konkret: Entbüroner Partnerschaft verteilt wird. Heute sind häufig beide Partner in sehr fordernden beruflichen Positionen tätig, entsprechend hat die «Paararbeitszeit» zugenommen. Das hat zu einem spannenden Phänomen geführt: Trotz hoher Belastung sehen sich Jüngere häufig dem Vorwurf konfrontiert, sie würden weniger Einsatzbereitschaft zeigen als ihre älteren Kollegen.

Im Positionspaper des BJÄ zur Arbeitsverdichtung schreiben Sie, Krankenhäuser würden zunehmend als Profitzentren angesehen werden, was starke Nachteile für das dort angestellte medizinische Fachpersonal, aber auch für die Patienten nach sich zieht. Wie kommt das? Wie wird diese Denkweise angefeuert?

Ein Kernproblem besteht darin, dass die Bundesländer ihrer Investitionsverpflichtung nicht ausreichend nachkommen. Das zwingt die Krankenhäuser dazu, aus den DRG-Fallpauschalen, die ja auf einer Ist-Kosten-Rechnung der für die Patientenversorgung notwendigen kratisierung, exzellentes Prozessmanagement und Delegation von nicht-ärztlichen Tätigkeiten im Beruflichen und von häuslichen Alltagsarbeiten im Privaten, damit in der knappen Freizeit der Fokus auf die Familie, Freundschaften und Hobbys gelegt werden kann.

Wie wird sich die Patientenversorgung in den nächsten 10 bis 20 Jahren verändern? Was muss im Gesundheitssystem geändert werden, dass immer noch eine gute medizinische Versorgung gewährleistet werden kann?

Ich bin zuversichtlich, dass wir in Zukunft durch weitere Innovationen unseren Patienten eine noch deutlich bessere Gesundheitsversorgung bieten können als heutzutage. Das wird aber nur gehen, wenn wir mutig sind neue Wege zu gehen. Das ist rechtlich, ethisch und medizinisch definitiv eine Herausforderung. Aber wir haben sicherlich keinen Grund, sorgenvoll in die Zukunft zu schauen. 
Im Masterplan «Medizinstudium 2020» soll gesichert werden, dass der akademische Aspekt des Medizinstudiums in Zukunft stärker betont wird. Insbesondere sollen so Dissertationen wieder eine höhere Qualität erreichen. Wie bewerten Sie diese Ansätze? Welche Änderungen am Medizinstudium halten Sie darüber hinaus für wichtig?

Hinter dem Masterplan «Medizinstudium 2020» steht eine politische Agenda, die ich sehr befremdlich finde. Im Kern geht es darum, die hausärztliche Versorgung dadurch zu stärken, dass die Zulassungsbedingungen und Inhalte des Medizinstudiums verändert werden. Das entspricht nicht meiner Vorstellung einer qualitätsorientierten Ausbildung und ist ein bildungspolitischer Prinzi- pienbruch. Ungeachtet dessen braucht eine wissenschaftlich basierte Medizin aber natürlich wissenschaftlich versierte Ärzte. Sinkende Promotionsquoten sind ein klares Indiz dafür, dass ein «Weiter so» der falsche Weg ist. Aber mit strukturellen Reformen die sicherlich an der Zeit sind - ist es nicht getan. Wir brauchen wieder Lust auf Forschung, Fortschritt und Veränderung!

\section{Dr. Schulte, vielen Dank für das offene Gespräch!}

Kontaktadresse: Dr. med. Kevin Schulte, Sprecher Bündis JUNGE ÄRZTE, Klinik für Innere Medizin IV, Nieren- und Hochdruckkrankheiten, Universitätsklinikum Schleswig-Holstein, Schittenhelmstraße 12, 24105 Kiel, Deutschland, kevinschulte@gmx.de.

\section{Die nächste Ausgabe Karger Kompass Dermatologie mit dem Themenschwerpunkt «Pädiatrische Dermatologie» erscheint am 13. April 2017!}

